\title{
Validación de cuestionario sobre percepciones y creencias de epidemia. Una estrategia para aprendizaje en el aula Validation of a questionnaire on perceptions and beliefs about the epidemic. A strategy for learning in the classroom
}

\author{
Rafael Tuesca Molina ${ }^{1}$, Nuria Rodríguez Ávila ${ }^{2}$, Carolina Moreno Castro ${ }^{3}$ \\ rtuesca@uninorte.edu.co; nrodriguez@ub.edu, carolina.moreno@uv.edu \\ ${ }^{1}$ Departamento de Salud Pública. Universidad del \\ Norte. Barranquilla. Colombia \\ ${ }^{2}$ Departamento de Sociología y Análisis de las \\ Organizaciones. Universidad de Barcelona. \\ Barcelona. España.
}

${ }^{3}$ Departamento de Teoría de los Lenguajes y Ciencias de la Comunicación. Universidad de Valencia. Valencia. España

\begin{abstract}
Resumen- El presente trabajo ofrece una validez de constructo de un cuestionario sobre percepciones y creencias de epidemia. El instrumento a utilizar se apoya con el uso del cine para favorecer aprendizaje significativo y fomentar aprendizaje a lo largo de la vida. Se aprecian dificultades en aspectos de comunicación y ética frente a una epidemia. Los resultados obtenidos en este piloto permiten afirmar que esta experiencia sirve para crear y desarrollar competencias profesionales de tipo comunicativa y pensamiento crítico. Se identificaron situaciones éticas en sucesos complejos que afectan el desempeño de su actividad profesional
\end{abstract}

Palabras clave: medios de comunicación, epidemias, aprendizaje, educación basada en competencias

\begin{abstract}
The present work offers a construct validity of a questionnaire about perceptions and beliefs of epidemic. The instrument to be used is supported by the use of cinema to promote meaningful learning and to encourage lifelong learning. There are difficulties in communication and ethics in the face of an epidemic. The results obtained in this pilot allow to affirm that this experience serves to create and to develop professional competences of type communicative and critical thinking. Ethical situations were identified in complex events that affect the performance of their professional activity
\end{abstract}

\section{Keywords: communications media, epidemics, learning,} competency-based Education

\section{INTRODUCCIÓN}

El cine facilita el aprendizaje, transforma el escenario pedagógico, posiciona la cultura, la comunicación y favorece la vivencia de forma integral al sujeto que aprende (Tornel., 2007; de la Torre et al., 2003-2004; Pérez., 2012; Loscos et al., 2006; González., 2009; Pardo \& Olaya., 2014; García et al., 2002). Aspectos interesantes de esta herramienta, corresponde con: abordar aspectos curriculares ocultos, visibilizar problemas complejos para incorporar y aplicar la investigación epidemiológica de una epidemia, reflexionar acerca del comportamiento social ante una situación emergente, visibilizar el trabajo multidisciplinar en contexto, ahondar en la dinámica y actuación de los medios de comunicación, reflexionar con la óptica de la autoridad sanitaria y sopesar aspectos de ética.(Andrade., 2013; Gómez., 2014). Esta conjugación de fenómenos permite que el estudiante elabore un análisis profundo de la naturaleza y del hombre frente a las amenazas que se expone y genera en un mundo global. Este fenómeno guarda estrecha relación con sucesos ambientales que favorecen brotes o epidemias, (Rodríguez., 2011; Augsburger., 2007; Martínez., 2007)

El estudio de un brote o epidemia requiere desarrollar diversas habilidades del estudiante $\mathrm{y}$ del profesional de salud. Dentro de estas habilidades se requiere la capacidad de dar unas respuestas adaptadas al contexto donde ocurren brotes a fin de minimizar riesgos e impactos. Los centros de vigilancia y de control de enfermedades tienen establecido una metodología epidemiológica positivista que involucra pasos estructurados. Estos elementos se sintetizan en: definición del caso, confirmación del caso, estimación de hipótesis provisionales del caso, desarrollo de la metodología descriptiva para el estudio epidemiológico (persona, tiempo y lugar), establecimiento de medidas de control, verificación de hipótesis y evaluación de las medidas de control con el objeto de generar lecciones aprendidas, evitar la diseminación y ahondar el mayor conocimiento de la historia natural y social de la enfermedad (Junta de Andalucía., 1988; Ministerio de Salud-Perú., 2003; OMS, 1993).

Se ha observado que estudiantes del área de salud identifican fácilmente elementos que conocen y saben ante un caso de brote o epidemia, mientras que cuando se sale de este contexto y se les proporciona ejercicios como el análisis de un material audiovisual les plantea un mayor desafío e interpretación. Exponer a un estudiante ante una película de epidemia, surgen dificultades para identificar y analizar 
elementos comunicacionales, éticos y culturales. Usualmente se identifican respuestas erróneas y esta situación obedece probablemente al enfoque formativo de los estudiantes del área de salud bajo el contexto biologicista de forma exclusiva (Loscos et al., 2006; Iscart-Isern et al., 2013). Otra dificultad en el aula para analizar aspectos comportamentales y éticos está relacionado con el escaso espacio que se destina a la reflexión, análisis y debate de aspectos de carácter epistemológicos propios de epidemiología. (Segura del Pozo., 2006)

El cine como actividad pedagógica propone una alternativa novedosa para que el docente posibilite diversos modelos de aprendizaje y desarrolle estrategias como el aprendizaje inclusivo, aprendizaje para la vida, entre otras. Se resalta posicionar el cine como hecho y bien cultural. El desarrollo de la didáctica para el aprendizaje utilizando el cine, requiere la construcción de los siguientes aspectos por parte del docente: objetivo de aprendizaje, contextualización, ejercicios de previsionado y postvisionado, el rol de los actores, su conexión con el sujeto que aprende, el contexto, el tema, el guion y otros elementos propios del séptimo arte. Algunos autores consideran que esta experiencia en el ámbito educativo refleja las dificultades habituales de implementar una estrategia pedagógica, por ejemplo: objetivos de aprendizaje concretos, manejo del tiempo, organización institucional y dificultad de los alumnos para trabajar en equipos y a esto se le agrega un modelo para trabajar con películas. (Pardo G, Olaya B 2014) (Carracedo 2009), (Rajadell, N. Violant, V. Bordas, I. 2012), (Michel, J.J.Tomas, A.2016) (Abad D, Correa J, Espinosa A 2016), (Pac D, Garcia N 2013)

\section{CONTEXTO}

Aprendizaje basado en problemas y aprendizaje para la vida.

En Colombia, al igual que otros países de la región y del entorno europeo, la formación de estudiantes del área de la salud incluye el desarrollo de habilidades y la medición de las mismas. Se visionan desde el currículum, a partir de la experiencia del Proyecto Tuning en Latinoamérica el desarrollo y construcción de competencias de tipo comunicativa, ética y toma de decisión efectiva que se sustenten desde una visión políticamente correcta y socialmente aceptable para la sociedad. Por ello, estas actuaciones deben reflejar conocimiento y habilidades en los educadores y educandos. (Pinilla, A. 2012) (Zapata W. 2005) (Ospina, J., Manrique, F. y Martínez, A. 2012)

En la asignatura de epidemiología o de salud pública el tema de estudio: manejo de brote/epidemia o pandemias, reviste interés dado que involucra el desarrollo y adquisición de habilidades cognoscitivas y actitudinales. Desde la mirada estratégica de la Universidad del Norte, se direccionan acciones para el desarrollo de competencias sociales en los estudiantes, que favorezcan el mejoramiento permanente de sus habilidades comunicativas, su capacidad para relacionarse e insertarse en la sociedad como líderes sociales, empresariales y políticos (Uninorte. Plan de Desarrollo 2013)

Los profesionales que trabajan en el área de epidemiología y salud pública consideran que esta actuación frente a brotes $y$ epidemias representa un reto. El estudiante del área de la salud maneja fácilmente los aspectos biológicos fundamentados del modelo positivista tradicional de Flexner; pero los aspectos comportamentales y éticos reflejan baja resolución y frecuentemente es incapaz de resolver problemas complejos (Pinilla A 2011). Es necesario enfocar y contextualizar a los estudiantes que estamos frente a un mundo global. Aunado a esto, es necesario identificar que desde los contextos locales y las realidades de un entorno, dado el caso, Colombia, que experimenta una transformación social, sanitaria y política que la hace vulnerable ante amenazas y riesgos globales (Ospina, J., Manrique, F. y Martínez, A. 2012). Por lo tanto, todos somos vulnerables en algún momento de nuestras vidas ante una amenaza, un riesgo. Las conductas se mezclan con la cultura, la tecnología y las exigencias del mercado. Esta condición, nos ubica en un ambiente inseguro acompañados por una combinación de ansiedad, miedo e indiferencia frente a fenómenos globales que junto al cambio climático y otros fenómenos impactaran la salud y el bienestar.

La formación académica de los profesionales de salud en Colombia, privilegia la acumulación de conceptos abstractos frente a problemas concretos. Esta situación deja de lado que desde la academia se desarrollen habilidades procedimentales de tipo comunicativa especialmente, formación de valores y estrategias para pensamiento crítico con actitudes profesionales propias del área de la salud. El resultado es un aprendizaje de contenidos superficiales en el alumno y una reducción en la estructura de la evaluación docente. De ahí el interés de proponer esta estrategia pedagógica que transforme el aprendizaje con un enfoque cambiante para aprender de acuerdo al contexto, los valores y el modelo económico-social vigente (Pinilla, A. 2011-2012). Por lo tanto, conscientes de este reto se propone desarrollar, validar y adaptar esta estrategia como un escenario optativo que facilite la puesta en escena de una realidad visionada desde el séptimo arte que representa la cosmovisión de un país, que represente sus miedos, sus concepciones y actuaciones frente al caso concreto de exponerse ante una situación compleja de emergencia, desastre o una situación de pandemia.

La elección de esta herramienta, del uso del cine comercial bajo la estrategia pedagógica ORA -Observar, Reflexionar y Aplicar-, incorpora instrumentos que facilitan la identificación de percepciones y creencias ante una situación de epidemia/pandemia, permitirá escribir, interpretar, y transformar la realidad fomentando capacidad crítica para entender y actuar involucrando aspectos sociales, sanitarios, económicos y políticos, lo que supone que se deba trabajar con otros profesionales y no profesionales. 
Esta unidad de aprendizaje en la asignatura de epidemiología, intenta desarrollar los siguientes objetivos: 1) acercar a los estudiantes con los aspectos más humanos de la enfermedad que relacione la problemática social-económica y política de una epidemia/pandemia en comparación con otros tópicos que se abordan en epidemiología (enfoque de determinantes sociales), 2) favorecer un escenario de aprendizaje activo, significativo, lúdico y colaborativo, 3) diseñar una actividad que permita al educando la oportunidad de relacionar los conceptos aprendidos teóricamente con una simulación de tipo casi práctica, 4) favorecer la construcción de escenarios que propicien habilitar competencias comunicativas y/o éticas relevantes o significativas ante la presentación de situaciones complejas de epidemias/pandemias, y 5) crear y validar herramientas que pueda tener validez para los participantes interesados en estos temas. (Méndez-Domínguez 2016)

\section{DESCRIPCIÓN}

Estructura del guion (previsionado): introducción de la actividad

Titulo original “Contagion” (Contagio, en español). Dirección: Steven Soderbergh. País: Estados Unidos. Año: 2011. Duración original: 106 minutos. Género: ciencia ficción-thriller-(historias cruzadas). Guión: Scott Burns. Distribuidora: Warner Bros. Pictures. Música: Cliff Martínez. Montaje: Stephen Mirrione. Reparto: Matt Damon, (Mitch Emhoff), Marion Cotillard (Dra. Leonora Orantes), Laurence Fishburne (Dr. Ellis Cheever), Jude Law (Alan), Gwyneth Paltrow (Beth Emhoff), Kate Winslet (Dra. Erin Mears), Chin Han (Sun Feng), Elliott Gould (Dr. Ian Sussman). Puntuación media de 5,8 a 6,8/10 (Méndez-Domínguez 2016)

Logística: Para llevar a cabo esta experiencia tipo pilotaje y validación se contó con el apoyo científico, logístico y financiero del programa de movilidad del profesorado de la Universidad de Barcelona. Para este desarrollo, se conformó un grupo de participantes voluntarios: estudiantes de sociología (4) y tres (3) profesionales expertos y con experiencia en el manejo de situaciones de amenazas, desastres y emergencias que han participado con otros profesionales de salud, políticos y gestores de salud. La actividad pedagógica se denominó: "Seminario científico. Análisis y herramientas de situaciones complejas”

Objetivo de aprendizaje: validar instrumentos y la metodología de trabajo en el aula. Se analizó de manera crítica cualquier paso dentro de la ruta de aprendizaje. Cada apartado de la ruta disponía de un espacio para discusión. El proceso inició con la presentación de los participantes en donde expresaron su experticia frente a situaciones complejas de diversa índole: manejo de violencia para grupos vulnerables, preparación y entrenamiento frente a situaciones de emergencia, apoyo para grupos de socorro y situaciones de intervención laboral/empresarial.

Posterior a esta dinámica que rompió el hielo y aclimató a los actores al encuentro; se plantearon los objetivos centrales: analizar a partir de la película propuesta (“Contagion”) creencias y percepciones con respecto a la acción comunicativa ante una epidemia (confianza y veracidad) por parte de medios, autoridades sanitarias y personal de salud. Analizar creencias y percepciones frente a un brote epidémico/pandémico desde la mirada del fenómeno en los sujetos a partir de su condición de vulnerabilidad, los mecanismos de prevención y finalmente los aspectos éticos de manera individual y colectivo que se presenta en la película teniendo en cuenta la actuación de los servicios de salud y de las organizaciones sanitarias

\section{Instrumentos de análisis:}

(Cuestionario ad-hoc de percepción y creencias relacionado con uso de películas como estrategia pedagógica, confianza de la autoridad sanitaria y rol de medios e industria farmacéutica ante una situación de epidemia/pandemia.

Se elaboró un cuestionario ad-hoc con 20 preguntas tipo escala Likert para valorar percepción y creencias mediante un gradiente respuesta de 1 a 5 . Siendo 1 "Totalmente en desacuerdo" (TD) y 5 "Totalmente de acuerdo" (TA) mientras que la opción 3 "Ni acuerdo, $\mathrm{Ni}$ desacuerdo". Además, se incluyeron datos básicos relacionados con auto-clasificación de sexo, edad en años, nivel de formación, tipo de programa académico y conocimiento previo del concepto de epidemia y pandemia. Luego se diligenció la encuesta. Al finalizar la actividad, se explicó la siguiente sesión donde se ilustró el visionado de la película, los objetivos de la actividad a desarrollar y se esbozó la estrategia de análisis para observar, relacionar y aplicar aspectos claves de epidemiología, comunicación y ética en el material cinematográfico.

Luego de diligenciar la encuesta se procedió a valorar la utilidad de cada ítem, su objetivo, la construcción y el lenguaje empleado a fin de facilitar una interpretación homogénea para el contexto latinoamericano e hispano parlante. La siguiente fase se presentó el video adaptado de la película "Contagion” reeditado con una duración de 29 minutos respetando la línea de tiempo y secuencia de la película sin alterar el desarrollo de secuencias y sucesos claves que representan la estrategia de investigación científica de tipo epidemiológico que va desde el rumor hasta culminar con probar la hipótesis, introducir medidas de intervención y el cierre del método epidemiológico.

La segunda sesión del proceso académico y de validación contempló la identificación de hechos o eventos relacionados con comunicación en riesgo, aspectos éticos/político de una epidemia/pandemia y se analizó las situaciones cotidianas que se presentaron en situación de epidemia pasada en una institución sanitaria en el contexto español.

\section{Actividad de cierre y lecciones aprendidas:}

El siguiente paso que cerró el proceso de observación, análisis y reflexión mediante la aplicación de lo aprendido permitió abordar elementos cognitivos y actitudinales pedagógicos ante una situación compleja. A 
fin de estimar el posible efecto de la introducción del video en el formato de aprendizaje en el aula se diligenció el cuestionario post, en donde se indagó los mismos ítems al inicio de la actividad de la primera sesión. Se retroalimentó las respuestas de los participantes, lo que permitió reflexionar acerca de las posturas previas y posteriores a la intervención pedagógica y valorar el proceso de aprendizaje significativo.

\section{Aspecto metodológico empleado:}

Este proceso se realizó de manera cualitativa descriptiva. En el aspecto descriptivo se empleó la distribución frecuentista de los ítems de estudio al analizar las opciones de respuesta en la fase pre y post. Posterior a esta fase, se ajustaron los ítems del cuestionario y se organizaron las preguntas en bloques y se incorporaron adaptaciones para el contexto español y latinoamericano que se adjunta al final del manuscrito (ver anexo 1). No se estimaron diferencias estadísticas ni pruebas no paramétricas por ser un grupo focal.

\section{Resultados}

La mediana de edad de los participantes fue de 39 años. El $67 \%$ de participantes de sexo femenino y de manera similar esta proporción conocía la definición al igual que la diferencia entre epidemia y pandemia.

Al inicio de la actividad pedagógica los participantes expresaron con acuerdo parcial el hecho de considerar que este tipo de películas puede modificar la forma de entender el comportamiento de los científicos, así mismo, refleja un estereotipo muy alejado de los científicos, consideran que las epidemias son estrategias que se inventa desde la industria farmacéutica. Frente a las agencias de gobierno y de salud, consideraron que implementan o aplican medidas impopulares. Expresaron que las instituciones/ agencias de salud siempre mienten. Manifestaron que la comunidad debe participar y apoyar la toma de decisiones para ganar confianza en sus acciones. Un total acuerdo en la mitad de los participantes corresponde con el hecho de que la comunidad o población tiene responsabilidad en la aparición de fenómenos de epidemia y pandemia. Se identificó que la opinión de los participantes se divide en que la mitad considera que el cine muestra una realidad y el resto está en desacuerdo. De otra parte, expresaron parcialmente desacuerdo en utilizar una epidemia para justificar gastos de gobierno.

La actividad pos-test se observaron cambios favorables en la percepción de los participantes, por ejemplo: percepción positiva del uso del cine como estrategia pedagógica, se planteó que luego de ver esta película y reflexionar sobre comportamientos habituales o no percibidos como de riesgo: pasarse la mano por la cara luego de realizar alguna actividad, el hecho de no lavarse las manos o lavar frutas o verduras antes de comerlas, visibiliza la exposición al riesgo de enfermar. Por lo anterior, los participantes consideraron que se sensibilizaron hacia la conducta de prevención. El rol del epidemiólogo fue percibido de una forma real y ajustada a la realidad; es decir, se cambió el imaginario de un personaje aislado del contexto realizando experimentos o buscando microbios a un sujeto que busca respuestas teniendo en cuenta las necesidades y los problemas de una comunidad. Se mejoró la percepción en la responsabilidad de la comunidad frente a la aparición de epidemias y pandemias al igual que el efecto del cambio climático. Se mantuvo la percepción ante las agencias internacionales y nacionales en relación a su confianza y capacidad de resolución ante una epidemia/pandemia.

\section{CONCLUSIONES}

El grupo focal mediante la estrategia “ORA” analizó los hechos comportamentales con respecto al rumor y la respuesta de las personas ante una epidemia/pandemia. Se considera que los medios de comunicación visibilizan el rumor, la alerta y los casos fatales; sin embargo, direccionan al público hacia la curiosidad y no favorecen actuaciones de educación, prevención y comprensión frente a la complejidad del fenómeno (Rigau. J, Clark. G. 2005). Los participantes consideran una actuación figurativa de políticos y el efecto de la presión de la industria farmacéutica dibujan un panorama de baja credibilidad, que repercute en las agencias de control de salud (Torres A 2009). En la sesión de lecciones aprendidas se expresó que dicha actividad pedagógica favorece el desarrollo de pensar de manera crítica, lo que les permitió identificar situaciones complejas que pasan desapercibidas al ver una película comercial que aborda una situación de epidemia. La actividad les permitió entender los pasos de la estrategia epidemiológica. Se propuso ahondar a partir de la película en dar respuestas asertivas y políticamente correctas para la comunidad asumiendo las situaciones que exhibe la cinta. La agrupación y ajuste de términos en las preguntas del cuestionario posibilitan avanzar para la siguiente fase de validación del instrumento. Esta actividad lúdica involucra al participante en su proceso de aprendizaje y le otorga un valor a este proyecto pedagógico.

\section{AGRADECIMIENTOS}

A la Universidad de Barcelona por apoyo logístico, operativo y científico dentro del programa de movilidad del profesorado A2 al igual que a la Universidad del Norte.

\section{REFERENCIAS}

Abad, D. Correa, J. Espinosa, A. (2016) El cineclub Cinexkrúpulos como un ambiente de aprendizaje para la enseñanza de valores [Tesis]. Universidad Tecnológica de Pereira. Colombia

Andrade, B. et al. (2007). Cine y habilidades para la vida. Reflexiones y nuevas experiencias de educación para la salud, cine y mass media. Gobierno de Aragón-Dirección General de Salud Pública. 272. ISBN 978-84

$-8380-022-5$

Augsburger, Ana Cecília, Gerlero, Sandra Silvana, \& Taboada, Ernesto. (2007). La formación de 
posgrado en Epidemiología: estrategias pedagógicas de una experiencia. Interface - Comunicação, Saúde, Educação, 11(21),

131-142. https://dx.doi.org/10.1590/S1414-32832007000100012

Carracedo C.(2009). Diez ideas para aplicar el cine en el aula (Aplicación y experiencias didácticas). De: I Congreso de español como lengua extranjera en Asia-Pacifico (CE/LEAP). Manila. Filipina: 229-267

De la Torre, S. Oliver, C. Violant, V. Tejada, J. Rajadell, N. Griona, M. (2003-2004). El cine como estrategia didáctica innovadora. Metodología de estudio de casos y perfil de estrategia docentes. Contextos Educativos, 6-7,65-86

González-Blasco, P. S.P. Pinheiro, T.R. Ulloa-Rodríguez, M.F. y Angulo-Calderón, N.M. (2009). El cine en la formación ética del médico: un recurso pedagógico que facilita el aprendizaje. Pers. Bioét.2 (33),114-127

Gómez-Arevalo, J.A.(2014). Reflexiones en torno a la ecoetica y sus aportes a la época contemporánea. Rev.Latinoam.Bioet. 14(2), 66-79

Icart-Isern, M.T. Garrido-Aguilar, E. de Miguel-Cañas, S. (2013) Cine en enfermería escolar. Efectividad del taller "Cine y salud escolar". FEM, 16 (4), 239-244

Junta de Andalucía. Consejería de Salud y Servicios Sociales. (1988) Investigación de brotes epidémicos.46. ISBN 8450577942

Loscos, J. Baños,J.E. Loscos, F. y de la Cámara, J. (2006). Medicina, Cine y Literatura: una experiencia docente en la Universitat Autónoma de Barcelona. Rev Med Cine 2, 138-142

Martínez Cruz, Evelyn, Pérez Cárdenas, Marcelino, \& Díaz LLanes, Guillermo. (2007). Estado actual en la formación académica de posgrado en salud pública en hispanoamerica. Revista Habanera de Ciencias Médicas, 6(4) Recuperado en 10 de enero de 2017, de http://scielo.sld.cu/scielo.php?script=sci_arttext\&pid=S1 729-519X2007000400006\&lng=es\&tlng=es.

Méndez-Domínguez, N. y Rodríguez-Castellanos, A. (2016). Pertinencia del análisis de la película contagio (2011) en el aprendizaje de la metodología clínica y epidemiológica en medicina. Rev Med Cine, 12(3):147155

Michel, J.J. Tomas, A.(2016) ¿Cómo leer un film? La formación ética a través del cine y la virtualidad. Informática na Educação: teoria e prática, 19 (1),6983

Ospina, J. Manrique-Abril, F. y Martínez-Martín, A. (2012). La formación de médicos generales según los requerimientos del sistema general de seguridad social en salud en Colombia. Revista Colombiana de Anestesiología. 40(2):124-126. https://doi.org/10.1016/S0120-3347(12)70025-4

Organización Mundial de la Salud. (1993) Investigación de brotes de enfermedades ambientales. Manual de entrenamiento. Ginebra, Suiza. OMS, 1993. OMS/PEP/91.35
Pac, D. García, N. (2013). El cine como herramienta de aprendizaje en el aula. Claves de una experiencia docente multidisciplinar en el ámbito económico. Revista Internacional de Organizaciones.10:181197

Pardo, G y Olaya B.(2014). Uso de la película como estrategia didáctica en una clase de educación artística (un estudio de caso). Actas Icono 14, 12, 396-408. (VII Simposio Las Sociedades Ante el Reto Digital). ISBN: 978-84-15816-10-2

Pérez, S. (2012). El cine como propuesta didáctica para la enseñanza de la música. Revista Galego-Portuguesa de Psicoloxia e Educación, 20(1),1138-1663

Pinilla, A. (2012) Aproximación conceptual a las competencias profesionales en ciencias de la salud.Rev. Salud pública. 14 (5): 852-864, 2012.

Pinilla, A. (2011). Modelos pedagógicos y formación de profesionales en el área de la salud. Acta Medica Colombiana, 36(4), 204-218. Retrieved June 01, 2017, from http://www.scielo.org.co/scielo.php?script=sci_arttext\& pid=S0120-24482011000400008\&lng=en\&tlng=es.

Rajadell, N. Violant, V. Bordas, I.(2012) Una semana de cine formativo como estrategia de simulación para un aprendizaje inclusivo desde la transdisciplinariedad y la globalidad. Quaderns Digitals. Ed 71.

Rodríguez Alonso, Beatriz, Fariñas Reinoso, Ana Teresa, Pérez Maza, Benito, Uranga Piña, Rolando, Alonso Uría, Rosa María, \& Morales Suárez, Ileana. (2011). Análisis del programa de formación del especialista en Higiene y Epidemiología en ensayos clínicos. Educación Médica Superior, 25(2), 17-28. Recuperado en 10 de enero de 2017, de http://scielo.sld.cu/scielo.php?script=sci_arttext\&pid=S0 864-21412011000200002\&lng=es\&tlng=pt

Rozo, R. \& Escobar, R. (2011). La educación médica en Colombia. Revista Med, 19(1), 7-9. Retrieved June 01, 2017, from http://www.scielo.org.co/scielo.php?script=sci_arttext\& pid=S0121-52562011000100001\&lng=en\&tlng=es

Rigau-Perez, J. y Clark, G (2005). Cómo responder a una epidemia de dengue: visión global y experiencia en Puerto Rico. Revista Panamericana de Salud Pública, 17(4), 282-293. https://dx.doi.org/10.1590/S1020-49892005000400012

Tornel, J.L. (2007). Cine formativo mediante enseñanza por tareas: Milagro en Milan. Escuela Abierta,10, 273-293

Torres, A. (2009). Algunas reflexiones sobre la gripe que no quieren llamar porcina: la visión desde Europa. Arch Bronconeumol. 45(7):315316.https://dx.doi/10.1016/j.arbres.2009.06.001

Universidad del Norte. Plan de Desarrollo 20132017.Educar para Transformar. (2013). Ediciones Uninorte. Barranquilla. 114p 
Zapata,W.A.S.(2005).Formación por competencias en educación superior. Una aproximación conceptual a propósito del caso colombiano. Revista iberoamericana de educación, 36(9), 1

Anexo. 1. Cuestionario de percepción y creencias acerca de epidemias y pandemias

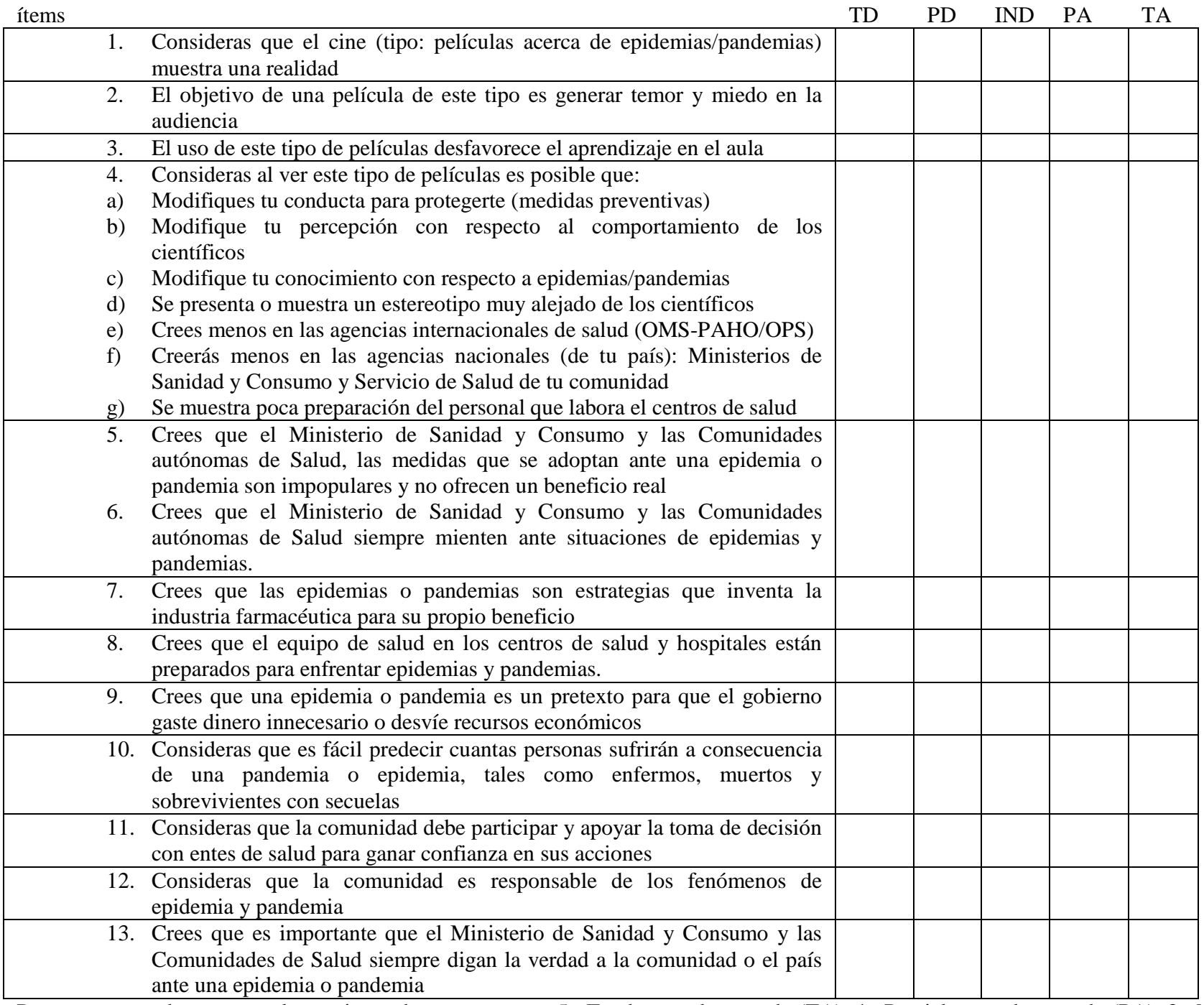

Para este grupo de preguntas las opciones de respuesta son: 5= Totalmente de acuerdo (TA); 4= Parcialmente de acuerdo (PA); 3= Ni acuerdo ni desacuerdo (IND; indiferente); 2: Parcialmente desacuerdo (PD) y 1= Totalmente en desacuerdo (TD) 